\title{
AVALIAÇÃO DA RUGOSIDADE SUPERFICIAL DE RESINAS ACRÍLICAS QUIMICAMENTE ATIVADAS E BISACRÍLICA.
}

\author{
EVALUATION OF SURFACE ROUGHNESS OF CHEMICALLY ACTIVATED ACRYLIC \\ RESIN AND BISACRYL
}

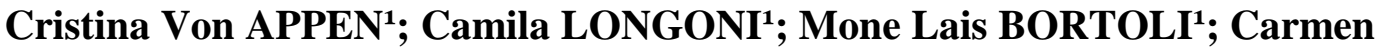 \\ Beatriz Borges FORTES ${ }^{2}$; Jefferson Tomio SANADA ${ }^{3}$
}

\author{
${ }^{1}$ Graduação em Odontologia, Faculdade de Odontologia \\ da UFRGS, Porto Alegre -RS \\ ${ }^{2}$ Mestre em Odontologia e Doutora em Ciências dos \\ Materiais, Professora Adjunta da Faculdade de \\ Odontologia da UFRGS, Porto Alegre - RS \\ ${ }^{3}$ Mestre e Doutor em Odontologia, Professor Adjunto da \\ Faculdade de Odontologia da UFRGS, Porto Alegre - \\ RS.
}

Correspondência:

Nome: Jefferson Tomio Sanada

E-mail: jefferson.sanada@ufrgs.br

Endereço: Faculdade de Odontologia - UFRGS Ramiro Barcelos, 2492 - Bairro Santana- Porto Alegre- CEP 90035-003

\section{Resumo}

Objetivo: Este trabalho avaliou a rugosidade superficial de uma Resina Acrílica Ativada Quimicamente (RAAQ) e uma Resina Bisacrílica, em diferentes técnicas de manipulação, para confecção de coroas provisórias.

Materiais e Métodos: Foram confeccionadas matrizes de silicone $(20 \times 10 \times 3 \mathrm{~mm})$ que serviram para confecção dos corpos de prova, que

formaram 4 grupos: Grupo Panela, Grupo Pincel, Grupo Fabricante e Grupo Bisacrílica $(n=12)$. Após a polimerização, foram realizadas 3 leituras longitudinais e 3 transversais com auxílio de um rugosímetro digital. As leituras foram repetidas após acabamento e polimento dos corpos de prova em Politriz Metalográfica. Os valores de rugosidade foram somados e criada uma média para cada corpo de prova, os quais foram somados novamente dentro do seu grupo criando uma média e desvio padrão, antes e após o polimento. Os dados foram submetidos a ANOVA a 2 critérios e Sidak's multiple comparisons test $(\mathrm{P}<0,05)$.

Resultados: Observou-se que há influência da técnica de manipulação e do tipo de resina acrílica na propriedade de rugosidade superficial. Dentre os grupos, Bisacrílica demonstrou menores valores de rugosidade, porém os outros grupos demonstraram uma melhora da rugosidade superficial após o polimento.

Conclusão: Há uma influência direta da técnica de manipulação e da marca comercial na propriedade de rugosidade superficial, demonstrando que existe a necessidade de um polimento para as resinas acrílicas possuírem uma rugosidade ideal, independente do tipo de técnica e marca comercial.

Palavras-chave: Coroas provisórias. Resinas acrílicas. Rugosidade superficial. 


\begin{abstract}
Objective: the aim of this study was to evaluate the surface roughness of Chemically Activated Acrylic Resin (CAAR) and Bysacril Resin used for temporary crown construction, with different techniques of manipulation.

Materials and methods: silicone matrixes $(20 \times 10 \times 3 \mathrm{~mm})$ were built and served for preparation of specimens, which formed 4 groups: Pressurized Pot Group, Bead Brush Group, Manufacturer's Group and Bisacryl Group $(\mathrm{n}=12)$. After polymerization, 3 longitudinal and 3 transversal readings were performed with the aid of a digital rugosimeter. The readings were repeated after finishing and polishing of the specimens in a Metallographic polishing machine. The roughness values were added and an average was created for each specimen, who was grouped again within each group creating an average and standard deviation, before and after they were polished. The data were submitted to a two-way ANOVA test and Sidak's multiple comparisons test $(\mathrm{P}<0.05)$.

Results: It was observed that there is influence between manipulation technique and type of acrylic resin on property of surface roughness. Among the groups, Bisacryl demonstrated smaller values of roughness, however other groups have shown an improvement of surface roughness after they were polished.

Conclusion: There is a direct influence between the technique of manipulation and commercial brand in the surface's roughness properties, showing that polishing is needed in order to acrylic resins achieve ideal roughness, independently of the chosen technique or commercial brand.
\end{abstract}

\title{
INTRODUÇÃO
}

A restauração com coroas provisórias é um componente crítico e uma das fases mais importantes do tratamento reabilitador protético com próteses fixas ${ }^{1}$. Dentro deste contexto, as coroas provisórias desempenham papéis importantes para a proteção pulpar e periodontal ao longo do tratamento. A proteção pulpar promove uma recuperação para a polpa após o preparo do dente e, além disso, promove um bom contorno e boa adaptação marginal que garantem o retorno à saúde de todos os tecidos moles traumatizados durante a preparação da coroa e de tomada de impressão ${ }^{2}$.

Existem muitos materiais disponíveis para restaurações provisórias e é fundamental que o profissional escolha o material que mais se aproxima das necessidades clínicas de cada paciente. De uma perspectiva histórica, as resinas acrílicas ativadas quimicamente (RAAQ) são os materiais mais antigos ainda em uso hoje ${ }^{3}$, porém com o avanço das tecnologias, as resinas bisacrílicas surgem no mercado como uma alternativa de material para restaurações provisórias.

Muitos dos problemas associados com resinas acrílicas tradicionais foram eliminados com os materiais bisacrílicos, que são fáceis de usar, flexíveis durante a inserção e remoção, radiopacos e possuem estabilidade de cor. Estes materiais são ideais para confecção de peças únicas e algumas situações com múltiplos elementos ${ }^{4}$.

As propriedades das resinas acrílicas são frequentemente avaliadas e relatadas na literatura. Propriedades como sorção e solubilidade, porosidade, resistência e módulo de flexão, microdureza, rugosidade e outras tantas propriedades mecânicas têm sido descritas ${ }^{5}$.

Uma das propriedades da resina acrílica que deve ser levada em consideração é a rugosidade superficial, pois interfere em um dos objetivos da coroa provisória, que é 
manter a saúde dos tecidos periodontais. Uma superfície rugosa não é apenas desconfortável para o paciente, como também favorece a aderência de alimentos e de biofilme dental. O ideal é que a superfície da resina tenha rugosidade abaixo do limiar de rugosidade estabelecido como ideal, que é de $0,2 \mu \mathrm{m}^{6,7}$.

Sabendo que a forma de manipulação e processamento das resinas acrílicas influenciam na rugosidade superficial ${ }^{8}$ e que o mercado possui diferentes marcas comerciais de diferentes qualidades, o objetivo deste estudo foi avaliar a rugosidade superficial de uma resina acrílica ativada quimicamente e uma resina Bisacrilica, utilizando diferentes técnicas de manipulação.

\section{MATERIAIS E MÉTODOS}

Para confecção das matrizes utilizadas na obtenção dos corpos de prova, foi utilizado um modelo em cera 7 com o formato retangular, medindo $20 \mathrm{~mm} \quad \mathrm{de}$ comprimento, $10 \mathrm{~mm}$ de largura e $3 \mathrm{~mm}$ de espessura. Posteriormente, esse modelo em cera foi envolvido por silicone de condensação de

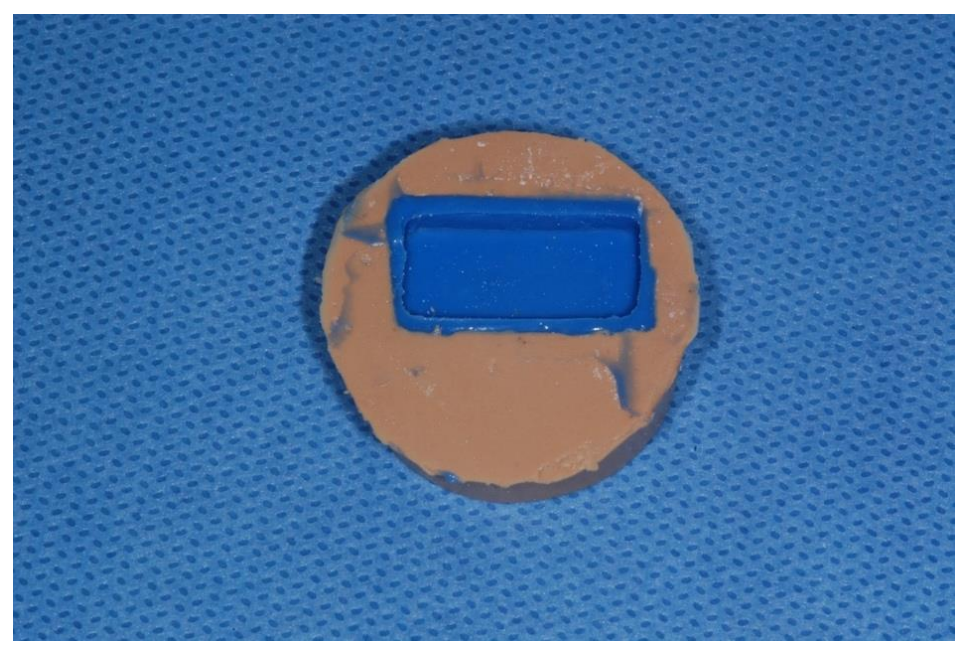
modo a formar um molde (matriz) a ser utilizado na confecção dos corpos (Fig. 1).

Foram utilizadas uma Resina Acrílica Ativada Quimicamente (RAAQ): Dencrilay (Dencril Comércio De Plásticos Importação E Exportação Ltda., Pirassununga - SP) e uma Resina Bisacrílica: Protemp ${ }^{\mathrm{TM}} 4$ (3M ESPE).

Os corpos de prova foram confeccionados de acordo com as 4 técnicas de manipulação da resina, formando assim os seguintes grupos:

- GRUPO PANELA- auto polimerização sob pressão em matriz de silicone;

- GRUPO PINCEL - auto polimerização utilizando a técnica do pincel;

- GRUPO FABRICANTE - auto polimerização utilizando a técnica da mistura em pote

Dappen;

- GRUPO BISACRÍLICA - auto polimerização utilizando sistema de automistura.

Para cada grupo foram confeccionados 12 corpos de prova baesado no trabalho de Nishida, Bianco, Hiramatsu, Moretti Neto, Rubo ${ }^{8}$.

No Grupo Panela, a resina acrílica foi devidamente misturada conforme as indicações do fabricante (proporção equivalente ao volume de 3:1 indicada pelo 
fabricante), sendo o polímero pesado com a ajuda de uma balança de precisão para utilizar 1,50g de pó a ser misturado com $0,70 \mathrm{ml}$ de monômero, medido através de uma pipeta. Em um pote Dappen, primeiramente foi despejado o líquido, seguido do pó, sendo então misturados suavemente por 5 segundos. Após devidamente manipulada, a resina foi inserida na matriz e esta posicionada contra uma plataforma de gesso e estabilizada com elásticos. O conjunto matriz + plataforma de gesso foi levado à polimerizadora sob pressão de $3 \times 105 \mathrm{~N} / \mathrm{m} 2$ por 15 minutos.

No Grupo Pincel, o monômero e o polímero foram distribuídos em potes tipo Dappen separados. Um pincel de uso rotineiro nas confecções de coroas provisórias teve sua extremidade umedecida com o líquido e colocada em contato com o pó; dessa maneira, as partículas de pó incorporadas pelo pincel umedecido formaram uma pequena esfera de massa de resina que foi levada ao interior da matriz de silicone.

No Grupo Fabricante, a obtenção dos corpos se deu com a saturação do conjunto líquido (monômero) + pó (polímero) em pote Dappen com imediato preenchimento da matriz, inserindo a resina ainda em sua fase arenosa.

Uma placa de vidro foi posicionada sobre a matriz até o final da polimerização.

No Grupo Bisacrílica, a resina foi inserida na matriz com o auxílio de um dispensador e pontas misturadoras fornecidas pelo fabricante.

Para o acabamento e polimento dos corpos de prova, foi utilizada uma Politriz Metalográfica (APL 4, Arotec, Cotia, SP), com dispositivos para polimentos múltiplos, capaz de realizar o polimento simultâneo em seis corpos de prova com refrigeração de água deionizada, proporcionando o paralelismo entre as superfícies polidas e a padronização dos espécimes.

A planificação das faces iniciou-se com lixa de silicone carbide de granulação 320 , seguido pelo polimento com as lixas de granulação 600 e 1200 . Foi utilizada a carga de $215 \mathrm{~g}$, durante 4 minutos, sempre com a politriz em alta velocidade (8).

Para não ter a influência dos grãos das primeiras lixas na qualidade do polimento das seguintes lixas, a cada troca de granulometria, os corpos de prova eram levados a uma cuba de Ultrassom Cristófoli (Cristófoli Equipamentos de Biossegurança, Campo Mourão, Paraná, Brasil), com frequência de $42 \mathrm{KHz}$, durante 2 minutos, com água deionizada e posteriormente eram secos com papel toalha.

A rugosidade de superfície ( $\mathrm{Ra}$ ) foi verificada através de um rugosímetro/perfilômetro Mitutoyo SJ-201 (Mitutoyo America Corporation, Aurora, Illinois, USA). Esse aparelho possui alta sensibilidade, com ponta apalpadora esférica de diamante, utilizado para medir rugosidade superficial quantitativamente. Sua ponta percorre a superfície e é acoplada a uma unidade que processa e interage a informação. O resultado é registrado de imediato por meio de um software.

O parâmetro $\mathrm{Ra}(\mu \mathrm{m})$ foi escolhido a fim de propiciar condições de comparação com resultados de outros estudos realizados, onde esta escala foi empregada, traduzindo o valor da média aritmética de todas as distâncias absolutas do perfil de rugosidade, dentro de extensão de medida Lm. Os parâmetros escolhidos foram: 
- T mínima $=2,5 \mathrm{~mm}$;

- $\mathrm{T}$ máxima $=2,5 \mathrm{~mm}$;

- $\mathrm{Lt}=4,80 \mathrm{~mm}$;

- $\mathrm{Lc}=0,800 \mathrm{~mm}$ (cut off);

$\cdot \mathrm{Lm}=4,55 \mathrm{~mm}$;

Sendo T a tolerância (valores extremos a serem considerados nas leituras), Lt o limite de tracejamento (extensão real percorrida pela ponta apalpadora), Lc o cut off (minimizando a interferência de ondulação da superfície) e Lm o limite de medição (extensão considerada na leitura).

Após obter os corpos de prova dos 4 grupos, foi realizada a medição da rugosidade superficial em dois momentos, antes e após o polimento. As mensurações foram feitas através de seis leituras por espécime, três no sentido longitudinal e três no sentido transversal. O valor de rugosidade superficial, de cada corpo de prova, foi obtido pela média aritmética das seis leituras e observou-se homogeneidade na amostra por meio do teste de normalidade de Shapiro-Wilko. Os valores foram submetidos ao teste estatístico de ANOVA a dois critérios e Sidak's de comparações múltiplas, com nível de significância de 5\%. As variáveis assumidas foram o tempo (antes e após polimento) e o tipo de técnica de manipulação. O programa estatístico utilizado foi o SYSTAT 13 (SYSTAT Software, San Jose, CA, USA).

\section{RESULTADOS}

Antes do polimento, o grupo do Fabricante apresentou os maiores valores de rugosidade superficial, seguida dos grupos Panela, Pincel e Bisacrílica, respectivamente (tabela 1).

Antes do polimento, todos os grupos foram estatisticamente diferentes (ANOVA) representadas pelas letras diferentes na coluna (tabela 1).

\begin{tabular}{|c|c|c|}
\hline Grupos & $\begin{array}{l}\text { Valores de Rugosidade Superficial } \\
\qquad(\mu \mathrm{m}) \text { antes do polimento }\end{array}$ & $\begin{array}{l}\text { Valores de Rugosidade Superficial } \\
(\mu \mathrm{m}) \text { após o polimento }\end{array}$ \\
\hline $\begin{array}{l}\text { Dencrilay } \\
\text { pincel }\end{array}$ & $1,44 \pm 0,14^{\mathrm{a} 1}$ & $0,21 \pm 0,05^{b 1}$ \\
\hline $\begin{array}{l}\text { Decrilay } \\
\text { fabricante }\end{array}$ & $2,43 \pm 0,28$ a2 & $0,26 \pm 0,10^{b 1}$ \\
\hline $\begin{array}{l}\text { Dencrilay } \\
\text { panela }\end{array}$ & $1,67 \pm 0,32$ a3 & $0,32 \pm 0,16^{b 1}$ \\
\hline Bisacrilica & $0,10 \pm 0,07^{a 4}$ & $0,15 \pm 0,06^{a 1}$ \\
\hline
\end{tabular}

Os grupos da marca comercial Dencrilay, após o polimento, não mostraram diferença estatisticamente significante quando comparados à marca comercial Protemp $^{\mathrm{TM}} 4$, com ou sem polimento, exceto quando o grupo Panela com polimento e Bisacrílica sem polimento são comparados. Após o polimento, não houve diferença estatisticamente significante entre os grupos Panela, Pincel e Fabricante (tabela 1). 
Bisacrílica foi o único grupo que apresentou uma média maior de rugosidade após o polimento, porém não foi estatisticamente significante, se mantendo dentro dos valores ideais $(0,2 \mu \mathrm{m})$, como mostra o tabela 1 .

\section{DISCUSSÃO}

Os resultados encontrados no presente estudo demostram que há influência da técnica de manipulação e do tipo de resina na propriedade de rugosidade superficial. Analisando os resultados encontrados antes do polimento dos corpos de prova, os grupos Pincel e Panela apresentaram valores de rugosidade semelhantes entre si e significativamente inferiores ao grupo Fabricante. Lee, Lai, $\mathrm{Hsu}^{9}$, relata que a rugosidade superficial é menor na técnica de pressão devido à diminuição do monômero residual. No entanto, esses baixos valores são superados pelo grupo pincel, pois, como descrito no trabalho de Nishida, Bianco, Hiramatsu, Moretti Neto, Rubo ${ }^{8}$, nesta técnica a polimerização ocorre em tempos diferentes para cada incremento de resina, o que leva a uma polimerização mais lenta e controlada, favorecendo assim a dissipação do calor da reação de polimerização em cada incremento e, consequentemente, diminuindo as chances de volatização do monômero.

Porém, os resultados de rugosidade superficial para os grupos pincel, panela e fabricante, antes do polimento, foram altos $(1,444-2,431 \mu \mathrm{m})$. Estes resultados corroboram com o estudo de Zissis, Polyzois, Yannikakis, Harrison ${ }^{10}$, que encontraram valores de rugosidade 3,4 a 7,6 $\mu \mathrm{m}$ antes do polimento.

Quirynen, Bollen ${ }^{11}$ relatam que superfícies rugosas facilitam a sobrevivência dos microrganismos na cavidade bucal, especialmente aqueles responsáveis por cáries e infecções periodontais, assim como também dificultam a remoção dos mesmos. Para que a superfície da resina acrílica esteja adequada para o uso na cavidade bucal ela deve receber um bom acabamento e polimento da superfície.

O grupo Bisacrílica foi o único que apresentou valores adequados de rugosidade superficial, que dever ser de $\mathrm{Ra}=0,2 \mu \mathrm{m}$, considerado ideal por diversos autores como Heath, Wilson ${ }^{12}$; Yamauchi, Yamamoto, Wakabayashi, Kawano ${ }^{13}$; Loney, Moulding, Hacker, Ritsco ${ }^{14}$; Leitão, Hegdahl ${ }^{15}$. Sugere-se que o aumento da rugosidade superficial após o polimento tenha ocorrido pela criação de ranhuras causadas pelo movimento constante das lixas durante o polimento dos corpos de prova em Politriz Metalográfica. De acordo com Guler, Kurt e Kulunk ${ }^{16}$, para o polimento de compostos bisacrílicos é recomendado o uso de pedra pomes e pasta de polimento. Outros autores sugerem que o polimento das resinas bisacrílicas deve ser realizado com algodão e álcool para remoção da camada de polimerização inibida pelo oxigênio, porém para não criarmos um viés (tipos de polimentos), optou-se por realizar o mesmo padrão de polimento a todos os grupos.

Segundo Leitão e Hegdahl ${ }^{15}$, logo após a confecção da peça de RAAQ a superfície não possui uma rugosidade superficial adequada. No entanto, MaalhaghFard, Wagner, Pink, Neme ${ }^{17}$, afirmam que isso pode ser alcançado se as coroas provisórias receberem um adequado acabamento e polimento da superfície, como pode 
ser observado nos grupos Panela, Pincel e Fabricante, que tiveram o valor de rugosidade superficial reduzidos após o polimento.

Se uma restauração provisória apresentar uma superfície bem polida, o manchamento de sua superfície, assim como o acúmulo de placa bacteriana, seriam reduzidos, diminuindo assim problemas estéticos e possíveis comprometimentos periodontais ${ }^{18}$.

\section{CONCLUSÃO}

Após a confecção de uma coroa provisória com RAAQ, esta não possui uma rugosidade superficial adequada, podendo prejudicar a saúde dos tecidos periodontais e assim, comprometer o tratamento reabilitador protético, porém após o polimento observa-se valores próximos aos ideais. O tipo de resina influenciou na propriedade de rugosidade superficial, sendo a marca Protemp ${ }^{\mathrm{TM}} 4$ a única que apresentou valores ideais de rugosidade tanto antes e após o polimento.

Há uma influência direta da técnica de manipulação e da marca comercial na propriedade de rugosidade superficial, demonstrando que existe a necessidade de um polimento para as resinas acrílicas alcançarem uma rugosidade ideal.

\section{REFERÊNCIAS}

1. Kim SH, Watts DC. Exotherm behavior of the polymer-based provisional crown and fixed partial denture materials. Dent Mater. 2004;20(4):383-7.

2. Strassler HE, Anolik C, Frey C. High-strength, aesthetic provisional restorations using a bis-acryl composite. Dent Today. 2007;26(11):128, 30-3; quiz 33, 3.

3. Emtiaz S, Tarnow DP. Processed acrylic resin provisional restoration with lingual cast metal framework. J Prosthet Dent. 1998;79(4):484-8.

4. Perry RD, Magnuson B. Provisional materials: key components of interim fixed restorations. Compend Contin Educ Dent. 2012;33(1):59-60, 2.

5. Mickeviciute E, Ivanauskiene E, Noreikiene V. In vitro color and roughness stability of different temporary restorative materials Stomatologija. 2016;18(2):66-72.

6. Garshasb M, Santos GC Jr, Rizkalla AS, Bohay R, Santos MJ. Effect of finishing procedures on the surface roughness of resin-modified glass-ionomer materials. Compend Contin Educ Dent. 2017 Jul;38(7):e1-e4.

7. Quirynen M, Bollen CM, Papaioannou W, Van Eldere J, van Steenberghe D. The influence of titanium abutment surface roughness on plaque accumulation and gingivitis: short-term observations. Int $\mathrm{J}$ Oral Maxillofac Implants. 1996;11(2):169-78.

8. Nishida CL, Bianco VC, Hiramatsu DA, Moretti Neto RT, Rubo JH. Análise da rugosidade superficial de diferentes marcas comerciais de resina acrílica para coroas provisórias. Full Dentistry in Science. 2011; 2:56-62. 
9. Lee SY, Lai YL, Hsu TS. Influence of polymerization conditions on monomer elution and microhardness of autopolymerized polymethyl methacrylate resin. Eur J Oral Sci. 2002;110(2):179-83.

10. Zissis AJ, Polyzois GL, Yannikakis SA, Harrison A. Roughness of denture materials: a comparative study. Int J Prosthodont. 2000;13(2):136-40.

11. Quirynen M, Bollen CM. The influence of surface roughness and surface-free energy on supra- and subgingival plaque formation in man. A review of the literature. J Clin Periodontol. 1995;22(1):1-14.

12. Heath JR, Wilson HJ. Surface roughness of restorations. Br Dent J. 1976;140(4):131-7.

13. Yamauchi M, Yamamoto K, Wakabayashi M, Kawano J. In vitro adherence of microorganisms to denture base resin with different surface texture. Dent Mater J. 1990;9(1):19-24.

14. Loney RW, Moulding MB, Hacker CH, Ritsco RG. Finishing and polishing of a poly (fluoroalkoxyphosphazene) resilient denture liner. Int J Prosthodont. 1994;7(4):362-7.

15. Leitão J, Hegdahl T. . On the measuring of roughness. Acta Odontol Scand. 1981;39(6):379-84.

16. Guler AU, Yilmaz F, Ural C, Guler E. Evaluation of 24-hour shear bond strength of resin composite to porcelain according to surface treatment. Int J Prosthodont. 2005;18(2):156-60.

17. Maalhagh-Fard A, Wagner WC, Pink FE, Neme AM. Evaluation of surface finish and polish of eight provisional restorative materials using acrylic bur and abrasive disk with and without pumice. Oper Dent. 2003;28(6):734-9.

18. Schwedhelm ER. Direct technique for the fabrication of acrylic provisional restorations. J Contemp Dent Pract. 2006;7(1):157-73. 